\title{
Metabolic and cellular analysis of alopecia in vitamin $D$ receptor knockout mice
}

\author{
Yoshiyuki Sakai, ${ }^{1}$ Jiro Kishimoto, ${ }^{2}$ and Marie B. Demay ${ }^{1}$ \\ ${ }^{1}$ Endocrine Unit, and \\ ${ }^{2}$ Cutaneous Biology Research Center, Massachusetts General Hospital, Harvard Medical School, Boston, Massachusetts, USA \\ Address correspondence to: Marie B. Demay, Endocrine Unit, Wellman 501, Massachusetts General Hospital, \\ 50 Blossom Street, Boston, Massachusetts 02114, USA. \\ Phone: (617) 726-3966; Fax: (617) 726-7543; E-mail: demay@helix.mgh.harvard.edu.
}

Jiro Kishimoto's current address is: Shiseido Research Center 2, Life Science Research Center, Yokohama, Japan.

Received for publication October 30, 2000, and accepted in revised form March 5, 2001.

Targeted ablation of the vitamin D receptor (VDR) results in hypocalcemia, hypophosphatemia, hyperparathyroidism, rickets, osteomalacia, and alopecia - the last a consequence of defective anagen initiation. To investigate whether the markedly elevated levels of 1,25 -dihydroxyvitamin $\mathrm{D}$ led to the alopecia, we raised VDR-null mice in a ultraviolet light-free environment and fed them chow lacking vitamin $\mathrm{D}$ for five generations. Despite undetectable circulating levels of 25-hydroxyvitamin D and 1,25-dihydroxyvitamin $\mathrm{D}$, alopecia persisted in the VDR-null mice, demonstrating that the alopecia was not secondary to toxic levels of 1,25-dihydroxyvitamin $\mathrm{D}$ interacting with an alternative receptor. Furthermore, alopecia was not seen in control littermates, suggesting that absence of ligand and absence of receptor cause different phenotypes. To identify the cell population responsible for the alopecia, we performed hair-reconstitution assays in nude mice and observed normal hair follicle morphogenesis, regardless of the VDR status of the keratinocytes and dermal papilla cells. However, follicles reconstituted with VDR-null keratinocytes demonstrated a defective response to anagen initiation. Hence, alopecia in the VDR-null mice is due to a defect in epithelial-mesenchymal communication that is required for normal hair cycling. Our results also identify the keratinocyte as the cell of origin of the defect and suggest that this form of alopecia is due to absence of ligand-independent receptor function.

J. Clin. Invest. 107:961-966 (2001).

\section{Introduction}

The biologically active metabolite of vitamin D, 1,25dihydroxyvitamin $\mathrm{D}$, interacts with target genes by binding to a nuclear receptor, the vitamin D receptor (VDR) (1). An animal model of hereditary 1,25-dihydroxyvitamin D-resistant rickets (HVDRR) generated by targeted ablation of the VDR in mice, results in hypocalcemia, hypophosphatemia, hyperparathyroidism, rickets, osteomalacia, and alopecia $(2,3)$. Normalization of mineral ion homeostasis by a diet high in calcium, phosphorus, and lactose normalizes this phenotype, with the exception of the alopecia (4). These results suggest that actions of the intact VDR (ligand dependent or ligand independent) are required for hair follicle homeostasis.

Hair follicle morphogenesis begins at embryonic day 14.5 in the mouse and is dependent on reciprocal interactions between the epithelium and the underlying mesenchyme. Several epithelially-derived signaling molecules have been shown to play a role in hair follicle morphogenesis $(5,6)$, including sonic hedgehog and bone morphogenetic proteins. The morphogenetic period extends until the third week of life, which marks the end of the first hair cycle. This first hair cycle, therefore, is unique and represents completion of embryological development of the hair follicles. Subsequently, the hair follicle undergoes cycling, a process that is regulated by epithelial-mesenchymal interactions (7). The hair cycle is characterized by a phase of hair growth (anagen) followed by a regression phase (catagen), a quiescent phase (telogen), and reentry into anagen to generate a new hair shaft (8). Despite substantial recent progress in identifying genes that play a role in hair follicle morphogenesis (5), identification of the factors that control postnatal hair cycling has remained elusive (7).

The VDR is expressed in the two major cell populations that comprise the hair follicle: the epidermal keratinocytes and the mesenchymal dermal papilla cells. (9). VDR expression in the hair follicle is increased during late anagen and catagen, correlating with decreased proliferation and increased differentiation of the keratinocytes (9). Our previous studies suggest that neonatal keratinocytes lacking functional VDRs possess the same proliferation and differentiation potential as wild-type keratinocytes (10). These studies, however, did not rule out a defect in the VDR-deficient keratinocytes related to their role in stimulating dermal papilla cells or receiving signals from these cells, interactions that are thought to be critical for the maintenance of the normal hair cycle. In fact, these studies demonstrated that hair follicle morphogenesis was normal, but there was a defect in anagen initiation after the morphogenetic period. 
Because 1,25-dihydroxyvitamin D downregulates its own biosynthesis by repressing the 25-hydroxyvitamin $\mathrm{D}-1 \alpha$-hydroxylase gene $(11,12)$ and increases its metabolism by upregulating the 24-hydroxylase gene through VDR-dependent actions $(13,14)$, VDR-ablated mice have very high levels of 1,25-dihydroxyvitamin $D$, even when mineral ion levels are normalized. Since 1,25-dihydroxyvitamin $\mathrm{D}$ has been shown to inhibit keratinocyte proliferation and to promote keratinocyte differentiation $(15,16)$, very high levels of this hormone or its metabolites may lead to alopecia by toxic interactions with an alternative receptor. To address the hypothesis that 1,25-dihydroxyvitamin D toxicity was the cause of the alopecia, we raised VDR-null mice in an ultraviolet light-free (UV-free) environment for five generations, on a diet lacking vitamin $\mathrm{D}$ metabolites.

In parallel, we also addressed the alternative hypothesis that the alopecia in the VDR-knockout mice was secondary to absence of hormone-dependent VDR actions, by examining the skin of wild-type mice with undetectable circulating levels of 25-hydroxyvitamin D and 1,25-dihydroxyvitamin D.

Since the VDR is widely expressed and is missing from all cells of the VDR-null mice, the organ and cell type in which VDR acts to maintain normal hair cycling is uncertain. To identify the cell population responsible for the defect and rule out a systemic cause for the alopecia, hair-reconstitution assays were performed in nude mice. Implantation of a mixture of keratinocytes and activated dermal papilla cells into a nude mouse host recapitulates the process of hair follicle morphogenesis (17), leading to a functional hair follicle. The use of activated (early anagen) dermal papilla cells is critical, since these cells do not retain their ability to induce the formation of functional hair follicles during all stages of the hair cycle. By implanting wild-type or VDR-null activated dermal papilla cells with wild-type or VDR-null keratinocytes, we were able to obtain hair follicles with epidermal and mesodermal components differing in VDR status (VDR ${ }^{+/+}$versus $\mathrm{VDR}^{-/-}$) and analyze which cell population was responsible for the defect in anagen initiation. Although epithelial cells are easily purified from the epidermis of neonatal mice, dissection of a pure population of dermal papilla cells is impracticable, since the dermal papilla is embedded in the ensheathing epidermal component. To overcome this problem, the VDRnull mice were crossbred with transgenic mice expressing green fluorescent protein (GFP) under the control of the versican promoter. In these transgenic mice, GFP is specifically expressed in the activated (anagen) dermal papilla cells (17); therefore, these cells are easily isolated from the dermis of neonatal mice by fluorescence-activated cell sorting. These studies enabled us to identify the cell responsible for impaired anagen initiation.

\section{Methods}

Animal maintenance. All studies performed were approved by the institutional animal care committee. VDR-null mice and control mice were maintained in a virus- and parasite-free barrier facility and exposed to a 12-hour light and 12-hour dark cycle. The heterozygous mothers and nude mice were fed autoclaved Purina rodent chow (5010; Ralston Purina Co., St. Louis, Missouri, USA) containing $1 \%$ calcium, $0.67 \%$ phosphorus, $0 \%$ lactose, and 4.4 IU vitamin $\mathrm{D} / \mathrm{g}$ (regular diet). Upon weaning at 18 days of age, VDR-null mice and control littermates were fed a $\gamma$-irradiated test diet (TD96348; Harlan Teklad, Madison, Wisconsin, USA) containing $2 \%$ calcium, $1.25 \%$ phosphorus, and $20 \%$ lactose supplemented with $2.2 \mathrm{IU}$ vitamin $\mathrm{D} / \mathrm{g}$, which has been shown to prevent abnormalities in mineral ion homeostasis in VDR-ablated mice (4). For generation of vitamin D-deficient animals, mice were maintained in a UV-free environment and fed a $\gamma$-irradiated vitamin $\mathrm{D}$-deficient diet (TD97340; Harlan Teklad), containing 2\% calcium, $1.25 \%$ phosphorus, and $20 \%$ lactose with 0 IU vitamin $\mathrm{D} / \mathrm{g}$. A Versican-GFP ${ }^{+/-}$male was bred to a VDR ${ }^{-/}$female to obtain Versican-GFP ${ }^{+/} / \mathrm{VDR}^{+/-}$offspring. Littermates were bred to obtain Versican-GFP ${ }^{+/-} / \mathrm{VDR}^{+/+}$and Versican-GFP ${ }^{+/-} / \mathrm{VDR}^{-/-}$pups for isolation of keratinocytes and dermal papilla cells.

Vitamin D assay. Circulating levels of 25-hydroxyvitamin $\mathrm{D}$ and 1,25-dihydroxyvitamin $\mathrm{D}$ were measured using radioimmunoassay kits (Nichols Institute, San Juan Capistrano, California, USA), following the manufacturer's instructions.

Isolation of keratinocytes and dermal papilla cells. Skin was dissected from the trunk of neonatal mice and floated on $0.25 \%$ trypsin (Life Technologies Inc., Grand Island, New York, USA) for $16-20$ hours at $4^{\circ} \mathrm{C}$. To obtain neonatal keratinocytes, the epidermis was then peeled off the dermis, minced extensively with scissors, and stirred in MEM with $4 \%$ Chelex-treated FCS (HyClone Laboratories, Logan, Utah, USA), epidermal growth factor (EGF) $(10 \mathrm{ng} / \mathrm{ml}$; Collaborative Research Inc., Cambridge, Massachusetts, USA) and

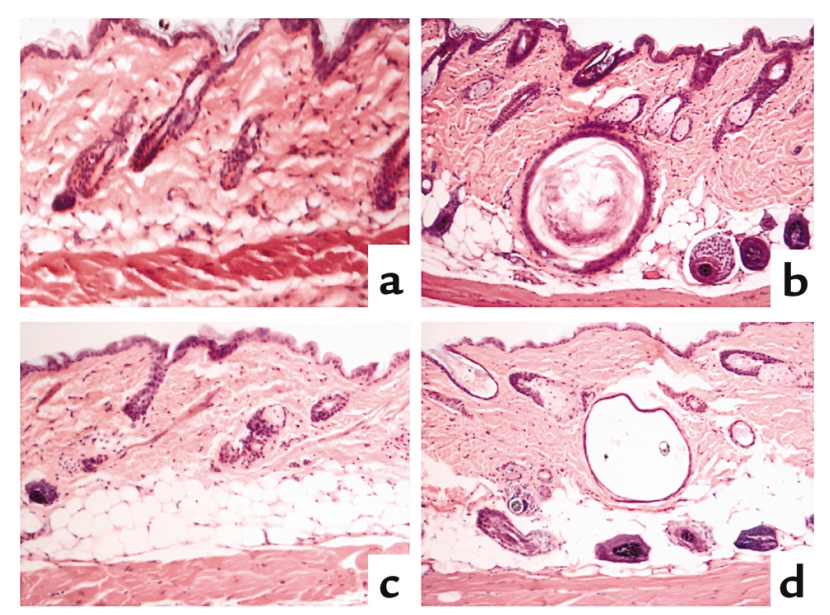

Figure 1

Histological analysis of the skin of 70-day-old normocalcemic VDRnull mice and control littermates. ( $\mathbf{a}$ and $\mathbf{c}$ ) Wild-type mice. (b and $\mathbf{d}$ ) VDR-null mice. ( $\mathbf{a}$ and $\mathbf{b}$ ) Vitamin $\mathrm{D}$ replete. ( $\mathbf{c}$ and $\mathbf{d}$ ) No detectable circulating levels of 25-hydroxyvitamin D or 1,25-dihydroxyvitamin D. 

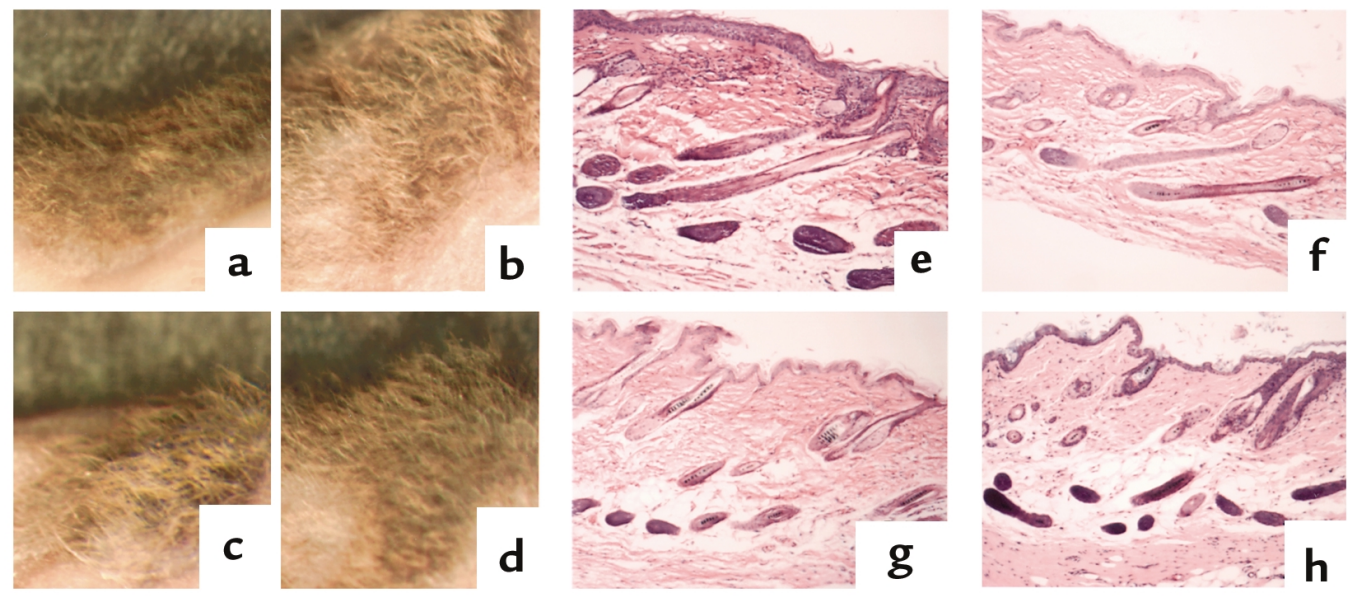

\section{Figure 2}

Hair-reconstitution assay 7 weeks after implantation of cells onto nude mice. (a-d) Gross view. (e-h) Hematoxylin and eosin staining of paraffin section. VDR status of cells implanted: (a and $\mathbf{e}$ ) wild-type keratinocytes, wild-type dermal papilla cells; (b and $\mathbf{f})$ VDR-null keratinocytes, wild-type dermal papilla cells; (c and $\mathbf{g}$ ) wild-type keratinocytes, VDR-null dermal papilla cells; ( $\mathbf{d}$ and $\mathbf{h}$ ) VDR-null keratinocytes, VDR-null dermal papilla cells.

$0.05 \mathrm{mM} \mathrm{CaCl}_{2}$ (low calcium medium) for 1 hour at $4^{\circ} \mathrm{C}(10)$. The resultant cell suspension was then filtered through a $70-\mu \mathrm{m}$ filter (Falcon, Franklin Lakes, New Jersey, USA) to remove clumps of cells and debris. The keratinocytes were pelleted by centrifugation for 5 minutes at room temperature. The remaining dermal layer was minced and incubated with $0.25 \%$ collagenase for 1 hour at $37^{\circ} \mathrm{C}$ with gentle stirring to dissociate cells. After filtration to remove debris $(70-\mu \mathrm{m}$ filter; Falcon), the cells were pelleted by centrifugation for 5 minutes at room temperature. Cell sorting was performed with a Moflo high-speed cell sorter (Cytomation Inc., Fort Collins, Colorado, USA) (17). Hair-reconstitution assays. GFP-positive dermal papilla cells $\left(5 \times 10^{6}\right)$ were pelleted with keratinocytes $\left(4 \times 10^{6}\right)$ and resuspended in $100 \mu \mathrm{l}$ of DMEM (Life Technologies Inc.) with $10 \%$ FCS. A $2.5-\mathrm{cm}$ incision was made on the flank of an athymic nude mouse $(n u / n u)$ to permit insertion of an implantation chamber specifically designed for these assays (18). This 2.5 -cm-diameter silicon chamber is engineered with a $0.5-\mathrm{cm}$ rim that remains in contact with the subcutaneous muscle (panniculus carnosus). The rim surrounds a $1-\mathrm{cm}$ open-bottomed dome through which the cells are implanted after the chamber is secured with surgical clips. To confirm that no cross-contamination of cell populations was present, control experiments were performed in which either GFP-positive dermal papilla cells or keratinocytes were implanted alone. The implantation chamber was removed after 1 week, and hair growth was assessed thereafter.

Anagen initiation. Under Avertin-induced general anesthesia, mice were subjected to depilation using Removal Wax Strips (Del Laboratories Inc., Farmingdale, New York, USA), following the manufacturer's instructions. Histological examination was performed 3 and 6 days after this procedure to assess keratinocyte proliferation (5-bromo-2'-deoxyuridine [BrdU] incorporation) and dermal papilla activation (GFP fluorescence) in response to anagen initiation.

Histology. Skin specimens were obtained from the middorsum of VDR-ablated mice and wild-type littermates or from the cell implantation site of the nude mice. After fixation for 3 hours in $4 \%$ formaldehyde in PBS ( $\mathrm{pH} 7.2$ ), specimens were processed, embedded in paraffin, and cut into 6- $\mu \mathrm{m}$ sections with a Leica RM 2025 microtome (Leica Microsystems Inc., Deerfield, Illinois, USA). For evaluation of GFP expression, tissue was embedded in Cryomatrix (Shandon Lipshaw, Pittsburgh, Pennsylvania, USA) on dry ice, immediately following which, 10$\mu \mathrm{m}$ sections were cut and fluorescence of the dermal papilla cells was assessed.

$B r d U$ incorporation. Mice were injected intraperitoneally with BrdU ( $250 \mathrm{mg} / \mathrm{kg}$; Sigma Chemical Co., Saint Louis, Missouri, USA) and 5-fluoro-2'-deoxyuridine (FdU) $(30 \mathrm{mg} / \mathrm{kg}$; Sigma Chemical Co.) and sacrificed 2 hours later. Skin specimens were obtained from the implanted region of nude mice, fixed for 3 hours in $4 \%$ formaldehyde in PBS ( $\mathrm{pH}$ 7.2), processed, embedded in paraffin, and cut into $6-\mu \mathrm{m}$ sections with a Leica $\mathrm{RM}$ 2025 microtome (Leica Microsystems Inc.). BrdU staining was performed using a BrdU Staining Kit (Zymed Laboratories Inc., South San Francisco, California, USA) following the manufacturer's instructions.

Alkaline phosphatase. Alkaline phosphatase (AP) staining was performed on frozen sections adjacent to those used to assess fluorescence (GFP activity). Staining was performed with an AP kit (Sigma Diagnostics, Inc., Saint Louis, Missouri, USA), following the manufacturer's instructions. Slides were then counterstained with nuclear fast red.

Statistical analyses. Data are presented as the mean \pm SEM. Student's unpaired $t$ test was used to identify significant differences $(P<0.05)$. 


\section{Results}

Effects of ligand deficiency. To rule out toxic effects of 1,25dihydroxyvitamin $\mathrm{D}$ as the cause of the alopecia, we raised VDR-null mice for five generations in a UV-free environment to prevent local production of vitamin $D$ metabolites in the skin. They were also fed a diet lacking vitamin $\mathrm{D}$ metabolites during this time. Despite undetectable levels of circulating 25-hydroxyvitamin D $(<1.2 \mathrm{ng} / \mathrm{ml})$ and 1,25 -dihydroxyvitamin $\mathrm{D}(<2.1 \mathrm{pg} / \mathrm{ml})$ in the fourth- and fifth-generation mice, alopecia, with dilatation of piliary canals and dermal cysts, persisted in the VDR-null mice (Figure 1d). These results indicate that toxic circulating levels of vitamin D metabolites do not play a critical role in the development of alopecia in VDR-null mice. Similarly, clinical and histological examination of the skin of wild-type littermates, raised under identical conditions, revealed that the absence of UV light exposure and of detectable circulating ligand had no effect on hair growth (Figure 1c). Combined with reports that failed to demonstrate alopecia in animal models of vitamin D deficiency or in patients and mice with 25 -hydroxyvitamin D $1 \alpha$-hydroxylase mutations (19), our data support the hypothesis that the pathogenesis of the alopecia in the VDR-knockout mice is secondary to the absence of receptor function. Furthermore, since absence of detectable circulating hormone is not associated with alopecia, it is probable that the actions of the VDR that are required for the maintenance of the normal hair cycle do not require hormone and are thus "ligand-independent" actions.

Hair-reconstitution assays. To rule out the possibility that the alopecia in the VDR-null mice is due to absence of the VDR in tissues other than skin and to identify the cell of origin of the defect, hair-reconstitution assays were performed. Keratinocytes and dermal papilla cells were isolated from Versican-GFP ${ }^{+/-} / \mathrm{VDR}^{+/+}$and Versican-GFP ${ }^{+/-} / \mathrm{VDR}^{-/-}$neonatal mice. At this stage, which still represents follicle morphogenesis, the hair follicles of the VDR-null mice are histologically normal (10). The proportion of dermal cells that were GFP positive (activated) was the same in neonatal VDR-null mice and control littermates $(38.2 \pm 2.0 \%$ vs. $39.3 \pm 3.4 \%$, respectively). The presence of normal numbers of activated dermal papilla cells, combined with the absence of detectable abnormalities in neonatal keratinocytes (10) in the VDR-null mice, further support the hypothesis that the VDR is not required for hair morphogenesis.

An incision was made in the flank skin of athymic nude mice to permit insertion of a silicon implantation chamber. Keratinocytes, either possessing or lacking the VDR, were then injected into the chamber, along with dermal papilla cells $\left(\mathrm{VDR}^{+/+}\right.$or $\left.\mathrm{VDR}^{-/}\right)$. To rule out cross-contamination of the keratinocyte and dermal papilla-cell populations or effects of the adjacent nude mouse cells, control experiments were performed using either dermal papilla cells alone or keratinocytes alone.

When keratinocytes were implanted together with dermal papilla cells, hair was observed within 3 weeks of implantation, regardless of the VDR status of the cells used (Figure 2, a-d). No histological difference in follicle formation was observed among the four combinations of cells used (Figure 2, e-h). These data confirm that the capacity for hair morphogenesis is preserved in both keratinocytes and dermal papilla cells lacking functional VDRs. When only keratinocytes or dermal papilla cells were implanted, no hair was observed, nor was there histological evidence of hair follicle formation (data not shown), confirming that no cross-contamination of cell populations had occurred.

Lack of VDR in keratinocytes is responsible for the defect in anagen initiation. Mice in which the hair-reconstitution assays were performed with wild-type keratinocytes maintained hair for more than 20 weeks after implantation, regardless of the VDR status of the dermal papilla cells used. In contrast, after 15 weeks, hair loss was seen in the mice in whom VDR-null keratinocytes were implanted, regardless of the VDR status of the dermal papilla cells. Twenty weeks after the reconstitution assay, there were no observable hair shafts in the implantation sites when VDR-null keratinocytes were used. This observation suggested that the lack of functional VDR in keratinocytes was responsible for the hair-cycle defect in the VDR-null mice.

We therefore tested the response of the reconstituted hair follicles to depilation and subsequent anagen initiation 7 weeks after implantation of cells. In hairreconstitution assays performed with wild-type keratinocytes and either wild-type or VDR-null dermal papilla cells, hair regrowth was observed within 2 weeks of depilation. In contrast, in assays performed with VDR-null keratinocytes and either wild-type or VDRnull dermal papilla cells, no hair regrowth was observed after depilation (Figure 3), even after 6 weeks of follow-
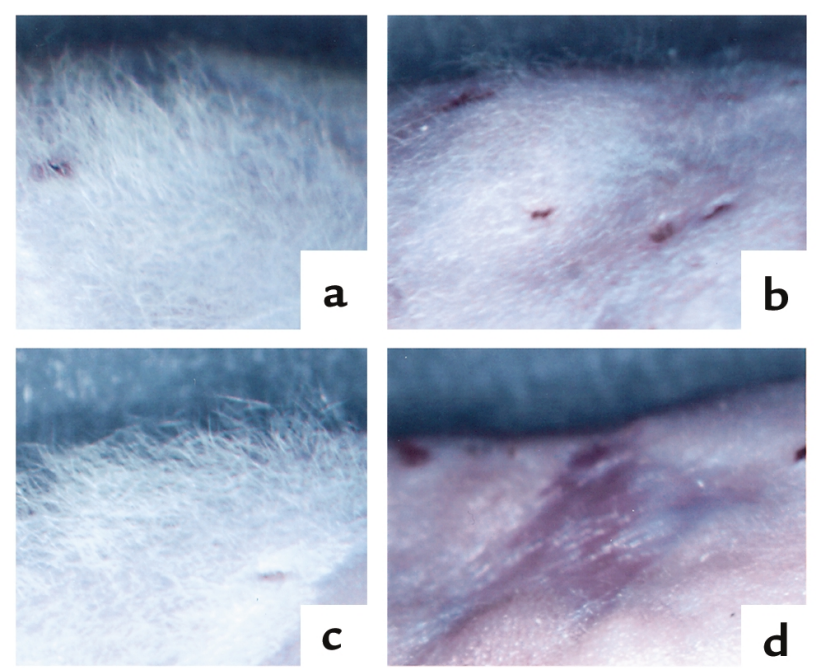

\section{Figure 3}

Response of reconstituted follicles to anagen initiation. Anagen was initiated 7 weeks after implantation of cells, and photographs were taken 3 weeks later. VDR status of cells implanted: (a) wild-type keratinocytes, wild-type dermal papilla cells; (b) VDR-null keratinocytes, wild-type dermal papilla cells; (c) wild-type keratinocytes, VDR-null dermal papilla cells; (d) VDR-null keratinocytes, VDR-null dermal papilla cells. 
up. This demonstrated that the $\mathrm{VDR}^{-/-}$keratinocytes were responsible for the failure of anagen initiation.

To examine whether the VDR-null keratinocytes were capable of responding to an anagen-initiating stimulus by increasing proliferation and activating dermal papilla cells, keratinocyte proliferation (assessed by BrdU incorporation) and dermal papilla activation (assessed by GFP) was evaluated 6 days after anagen initiation. In the reconstitution assays performed with wild-type keratinocytes, numerous anagen follicles containing BrdUpositive keratinocytes were visualized in the dermis. In contrast, in the assays performed using VDR-null keratinocytes, no anagen follicles were observed in the dermis, and BrdU staining was largely restricted to the epidermal keratinocytes and those lining the markedly dilated piliary canals (Figure 4, a-d). To examine whether activation of dermal papilla cells by the VDRnull keratinocytes was also impaired, dermal fluorescence was evaluated 3 days (data not shown) and 6 days after depilation (Figure 4, e-h). Since dermal papilla cells exhibit AP activity during the entire hair cycle (20) (and are the only positive follicle cells during early and mid-anagen), we performed AP staining on adjacent sections. In assays performed using wild-type keratinocytes and either wild-type or VDR-null dermal papilla cells, $\mathrm{AP}$ and GFP-positive signals colocalized in the region of the dermal papilla cells (Figure 4, e and g, arrows). However, when the reconstituted follicles were generated using VDR-null keratinocytes and dermal papilla cells of either genotype, AP-positive cells were observed without dermal fluorescence (Figure 4, $\mathrm{f}$ and $\mathrm{h}$ ).

\section{Discussion}

The association of VDR gene mutations with alopecia in both humans (HVDRR) (21) and mice, combined with the observation that VDR-ablated mice develop alopecia regardless of their mineral ion status (4), confirms that the hair follicle is an important target of VDR action. The experiments presented herein were designed to address three questions concerning the mechanism of the alopecia in the VDR-null mice: Is it caused by toxic levels of vitamin D metabolites? Alternatively, is it caused by absence of ligand-dependent activation of the VDR? In what cell does the VDR normally function to prevent the development of alopecia? At the age of 4 weeks, the $\mathrm{VDR}^{-/}$mice begin to develop perioral and periorbital alopecia. This hair loss progressed to involve the entire body over the next 3 months. Histologically, dilatation of piliary canals and large dermal cysts are evident by 7 weeks. Since the development of alopecia correlates temporally with a marked increase in circulating 1,25-dihydroxyvitamin D levels (3) and 1,25-dihydroxyvitamin $\mathrm{D}$ has been shown to have important biological effects on keratinocytes (15), toxic levels of this hormone or its metabolites may have a deleterious effect on hair cycling by interacting with an alternative receptor. However, our studies demonstrate that eliminating toxic circulating levels of 1,25-dihydroxyvitamin $\mathrm{D}$ does not prevent alopecia.

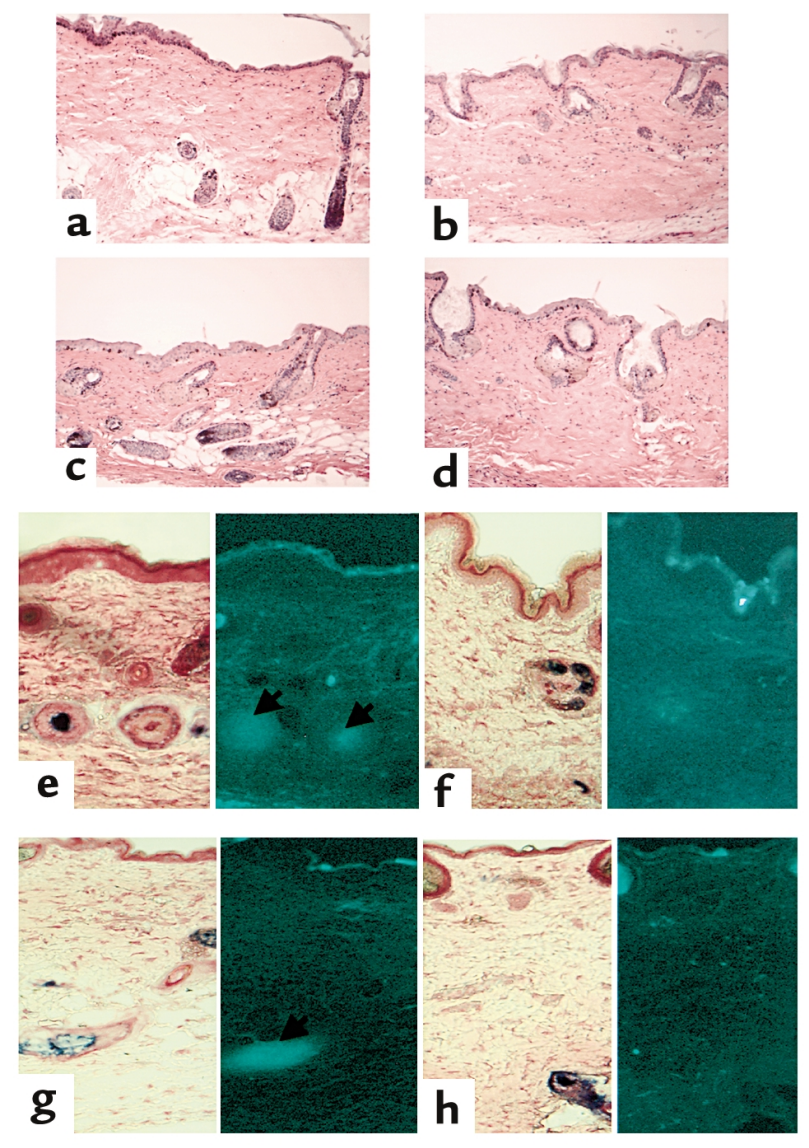

Figure 4

Response of reconstituted follicles to anagen 7 weeks after implantation of cells. Mice were sacrificed 6 days after anagen initiation, 2 hours after $\mathrm{BrdU}$ injection. (a-d) BrdU incorporation (dark brown) assessed on paraffin sections. (e-h) AP activity (blue stain) and GFP expression (e and $\mathbf{g}$, arrowheads). VDR status of cells implanted: (a and $\mathbf{e}$ ) wild-type keratinocytes, wild-type dermal papilla cells; (b and f) VDR-null keratinocytes, wild-type dermal papilla cells; (c and $\mathbf{g}$ ) wild-type keratinocytes, VDR-null dermal papilla cells; (d and $\mathbf{h}$ ) VDR-null keratinocytes, VDR-null dermal papilla cells.

The absence of alopecia in clinical vitamin D deficiency and in patients or mice with 25 -hydroxyvitamin $\mathrm{D}-1 \alpha$-hydroxylase mutations supports the hypothesis that the pathogenesis of the alopecia is a consequence of impaired receptor function rather than ligand deficiency. Nevertheless, neither of these observations eliminate the possibility that severe deficiency of 25 -hydroxyvitamin D or 1,25-dihydroxyvitamin D might cause alopecia because of impaired ligand-dependent VDR actions. In vitamin D deficiency, low circulating concentrations of 25-hydroxyvitamin D are seen (less than 4-5 ng/ml), and serum values of 1,25-dihydroxyvitamin D may be low, normal, or even elevated $(22,23)$. In 25 -hydroxyvitamin $\mathrm{D}-1 \alpha$-hydroxylase mutations, although 1,25-dihydroxyvitamin D serum levels are low, they are still detectable (greater than $10 \mathrm{pg} / \mathrm{ml}$ ) (19). Therefore, absence, rather than deficiency, of hormone or functional receptor might be required for 
alopecia. In wild-type mice maintained in a UV-free environment to prevent local vitamin $D$ synthesis, the presence of undetectable circulating levels of 25hydroxyvitamin $\mathrm{D}$ and 1,25-dihydroxyvitamin $\mathrm{D}$ did not lead to alopecia. This suggests that an alternative ligand exists for the VDR or that the alopecia in the VDR-null mice is secondary to lack of effects of the VDR that are not dependent on ligand.

In vitro data have demonstrated several biological effects mediated by unliganded nuclear receptors, including the closely related thyroid hormone receptors (TRs) $(24,25)$. TR isoforms exert both ligand-independent and ligand-dependent actions. Similarly, the unliganded VDR can repress basal transcription, and unliganded VDR/retinoid-x-receptor (VDR/RXR) causes VDR-mediated repression of basal transcription on DR3 response elements (26). TRs are thought to mediate ligand-independent repression on positive thyroid response elements (TREs) through the recruitment of corepressors and resulting histone deacetylase-containing complexes (27-29). Binding of the ligand results in release of the corepressor and recruitment of coactivators. Of note, one TR isoform, TR $\beta 2$, exhibits greater ligand-independent activation on TREs than other TR isoforms. This isoform binds a subset of nuclear-receptor coactivators in the absence of ligand, which may explain its ligand-independent effects (30). Interestingly, in vivo studies in mice lacking all known TRs demonstrate phenotypes distinct from those of thyroid hormone deficiency, supporting the hypothesis that the ligand-independent actions of TRs demonstrated in vitro are likely to have in vivo relevance (31). Similarly, our studies of VDR-null mice demonstrate a distinct phenotype from that of hormone deficiency. They suggest that the VDR has in vivo ligand-independent effects and that keratinocytes may express VDR coregulators in a developmental stage-specific fashion.

Using hair-reconstitution assays, we were able to reproduce the key features observed in the VDR-null mice: normal hair morphogenesis, gradual hair loss, impaired response to anagen initiation, and dilatation of piliary canals. These studies have permitted us to identify the keratinocyte as the cell responsible for the alopecia in the VDR-null mice. They demonstrate that the alopecia in the VDR-null mice is due to the inability of the VDR-null keratinocytes to proliferate in response to anagen initiation, as well as to activate dermal papilla cells (assessed by GFP fluorescence) in response to this stimulus. We also suggest that this defect may be due to absence of critical ligand-independent effects of the VDR in vivo.

\section{Acknowledgments}

We would like to express our appreciation to Henry M. Kronenberg for critically reviewing this article. This work was supported by NIH grant DK-46974 (to M.B. Demay)

1. Haussler, M.R., et al. 1998. The nuclear vitamin D receptor: biological and molecular regulatory properties revealed. J. Bone Miner. Res. 13:325-349.
2. Li, Y.C., et al. 1997. Targeted ablation of the vitamin D receptor: an animal model of vitamin D-dependent rickets type II with alopecia. Proc. Natl. Acad. Sci. USA. 94:9831-9835.

3. Yoshizawa, T., et al. 1997. Mice lacking the vitamin D receptor exhibit impaired bone formation, uterine hypoplasia and growth retardation after weaning. Nat. Genet. 16:391-396.

4. Li, Y.C., et al. 1998. Normalization of mineral ion homeostasis by dietary means prevents hyperparathyroidism, rickets, and osteomalacia, but not alopecia in vitamin D receptor-ablated mice. Endocrinology. 139:4391-4396.

5. Oro, A.E., and Scott, M.P. 1998. Splitting hairs: dissecting roles of signaling systems in epidermal development. Cell. 95:575-578.

6. Dlugosz, A. 1999. The Hedgehog and the hair follicle: a growing relationship. J. Clin. Invest. 104:851-853.

7. Stenn, K., and Paus, R. 1999. What controls hair follicle cycling? Exp. Dermatol. 8:229-236.

8. Paus, R., and Cotsarelis, G. 1999. The biology of hair follicles. N. Engl.J. Med. 341:491-497.

9. Reichrath, J., et al. 1994. Hair follicle expression of 1,25-dihydroxyvitamin D3 receptors during the murine hair cycle. Br. J. Dermatol. 131:477-482.

10. Sakai, Y., and Demay, M.B. 2000. Evaluation of keratinocyte proliferation and differentiation in vitamin $\mathrm{D}$ receptor knockout mice. Endocrinology. 141:2043-2049.

11. St-Arnaud, R., Messerlian, S., Moir, J.M., Omdahl, J.L., and Glorieux, F.H. 1997. The 25-hydroxyvitamin D 1-alpha-hydroxylase gene maps to the pseudovitamin D-deficiency rickets (PDDR) disease locus. J. Bone Miner. Res. 12:1552-1559.

12. Takeyama, K., et al. 1997. 25-Hydroxyvitamin D3 1alpha-hydroxylase and vitamin D synthesis. Science. 277:1827-1830.

13. Kerry, D.M., et al. 1996. Transcriptional synergism between vitamin Dresponsive elements in the rat 25-hydroxyvitamin D3 24-hydroxylase (CYP24) promoter. J. Biol. Chem. 271:29715-29721.

14. Ohyama, Y., et al. 1994. Identification of a vitamin D-responsive element in the $5^{\prime}$-flanking region of the rat 25-hydroxyvitamin D3 24-hydroxylase gene. J. Biol. Chem. 269:10545-10550.

15. Bikle, D.D., and Pillai, S. 1993. Vitamin D, calcium, and epidermal differentiation. Endocr. Rev. 14:3-19.

16. Eckert, R.L., Crish, J.F., and Robinson, N.A. 1997. The epidermal keratinocyte as a model for the study of gene regulation and cell differentiation. Physiol. Rev. 77:397-424.

17. Kishimoto, J., et al. 1999. Selective activation of the versican promoter by epithelial-mesenchymal interactions during hair follicle development. Proc. Natl. Acad. Sci. USA. 96:7336-7341.

18. Dotto, G., Weinberg, R., and Ariza, A. 1988. Malignant transformation of mouse primary keratinocytes by Harvey sarcoma virus and its modulation by surrounding normal cells. Proc. Natl. Acad. Sci. USA. 85:6389-6393.

19. Glorieux, F.H., and St-Arnaud, R. 1997. Vitamin D pseudodeficiency. In Vitamin D. D. Feldman, F.H. Glorieux, and J.W. Pike, editors. Academic Press. San Diego, California, USA. 755-764.

20. Handjiski, B.K., Eichmuller, S., Hofmann, U., Czarnetzki, B.M., and Paus, R. 1994. Alkaline phosphatase activity and localization during the murine hair cycle. Br. J. Dermatol. 131:303-310.

21. Malloy, P.J., Pike, J.W., and Feldman, D. 1999. The vitamin D receptor and the syndrome of hereditary 1,25-dihydroxyvitamin D-resistant rickets. Endocr. Rev. 20:156-188.

22. Pettifor, M.P., and Daniels, E.D. 1997. Vitamin D deficiency and nutritional rickets in children. In Vitamin D. D. Feldman, F.H. Glorieux, and J.W. Pike, editors. Academic Press. San Diego, California, USA. 663-678.

23. Walters, M., Kollenkirchen, U., and Fox, J. 1992. What is Vitamin D deficiency? Proc. Soc. Exp. Biol. Med. 199:385-393.

24. Horlein, A.J., et al. 1995. Ligand-independent repression by the thyroid hormone receptor mediated by a nuclear receptor co-repressor. Nature. 377:397-404.

25. Chen, J.D., and Evans, R.M. 1995. A transcriptional co-repressor that interacts with nuclear hormone receptors. Nature. 377:454-457.

26. Yen, P.M., Liu, Y., Sugawara, A., and Chin, W.W. 1996. Vitamin D receptors repress basal transcription and exert dominant negative activity on triiodothyronine-mediated transcriptional activity. J. Biol. Chem. 271:10910-10916.

27. Alland, L., et al. 1997. Role for N-CoR and histone deacetylase in Sin3mediated transcriptional repression. Nature. 387:49-55.

28. Heinzel, T., et al. 1997. A complex containing $\mathrm{N}-\mathrm{CoR}, \mathrm{mSin} 3$ and histone deacetylase mediates transcriptional repression. Nature. 387:43-48.

29. Nagy, L., et al. 1997. Nuclear receptor repression mediated by a complex containing SMRT, mSin3A, and histone deacetylase. Cell. 89:373-380.

30. Oberste-Berghaus, C., et al. 2000. Thyroid hormone-independent interaction between the thyroid hormone receptor beta 2 amino terminus and coactivators. J. Biol. Chem. 275:1787-1792.

31. Gothe, S., et al. 1999. Mice devoid of all known thyroid hormone receptors are viable but exhibit disorders of the pituitary-thyroid axis, growth and bone maturation. Genes Dev. 13:1329-1341. 